\title{
Nuclear multifragmentation induced by electromagnetic fields of ultrarelativistic heavy ions
}

\author{
I.A. Pshenichnov ${ }^{1,2} \dagger$, I.N. Mishustin ${ }^{2,3} \ddagger$, \\ J.P. Bondorf ${ }^{2}$, A.S. Botvina ${ }^{1,4}$, A.S. Iljinov ${ }^{1}$ \\ ${ }^{1}$ Institute for Nuclear Research, Russian Academy of Science, \\ 117312 Moscow, Russia \\ 2 Niels Bohr Institute, DK-2100 Copenhagen, Denmark \\ ${ }^{3}$ Kurchatov Institute, Russian Research Center, \\ 123182 Moscow, Russia \\ ${ }^{4}$ Hahn-Meitner-Institut, D-14109 Berlin, Germany
}

\begin{abstract}
We study the disintegration of nuclei by strong electromagnetic fields induced by ultrarelativistic heavy ions. The proposed multi-step model includes 1) the absorption of a virtual photon by a nucleus, 2) intranuclear cascades of produced hadrons and 3) statistical decay of the excited residual nucleus. The combined model describes well existing data on projectile fragmentation at energy $200 \mathrm{GeV}$ per nucleon. Electromagnetic multifragmentation of nuclei is predicted to be an important reaction mechanism at RHIC and LHC energies.
\end{abstract}

PACS: 25.70.De, 25.70.Pq, 25.75.-q

Key words: electromagnetic dissociation, relativistic heavy ions, intranuclear cascade, statistical multifragmentation model

†E-mail: pshenichnov@al10.inr.troitsk.ru

¥E-mail: mishustin@nbi.dk 


\section{Introduction}

Many experiments with relativistic heavy ions (RHI) are carried out now as well as planned for the future. In these experiments the main interest is focused on nucleus-nucleus collisions with small impact parameters corresponding to a significant overlap of nuclei. Central collisions are most appropriate for studying the highly excited and compressed hadronic matter and the quark-gluon plasma. Peripheral collisions are used to study the fragmentation of spectators.

However, the collisions with impact parameters exceeding the sum of nuclear radii, i.e. with no direct overlap of nuclei, can also significantly contribute to the nuclear reactions via the long-range electromagnetic interaction. The relativistic Coulomb excitation of nuclei was studied by many authors, see e.g. refs. [1, 2, 3]. This process is especially important in ultrarelativistic collisions with the Lorentz factors $\gamma \gg 1$. During a short time when colliding nuclei are close to each other, the potential of Lorentz-boosted Coulomb field [4] is very strong, $V_{c} \sim \alpha \gamma Z / b$, where $Z$ is the nuclear charge and $b$ is an impact parameter, $\alpha$ is the fine structure constant. For typical values $\gamma \gg \alpha^{-1}, Z \sim 50$ and $b \sim 10 \mathrm{fm}$ this potential $V_{c} \sim$ $\alpha \gamma \mathrm{GeV}$ considerably exceeds the nuclear forces and one can expect very dramatic phenomena. Among other interesting effects such an electromagnetic field will lead to the complete disintegration of nuclei, a phenomenon known already in nuclear reactions under the name of "multifragmentation" [5, 6]. The main aim of the present paper is to estimate the importance of these electromagnetic processes and to draw attention to a potentially rich area of research associated with the behaviour of nuclear matter under very strong electromagnetic forces.

As a first step we describe these reactions within a simplified approach known as the Weizsäcker-Williams method. According to this method one can represent the Lorentz-boosted Coulomb field as a pulse of radiation of virtual photons [2, 4]. Within this approach we use the virtual photon spectrum calculated for the pointlike charge distributions, that is justified only for collisions without direct nuclear overlap. As it was found in [7], the correction of the spectrum due to the finite sizes of colliding nuclei is of minor importance for the total fragmentation cross section.

The aim of the present paper is threefold. Firstly, we present a model of electromagnetic interaction of relativistic heavy ions which makes it possible to calculate the inclusive and exclusive characteristics of such interactions and compare them with existing experimental data. Secondly, we calculate the electromagnetic dissociation (ED) cross sections and the yields of hadrons and nuclear fragments produced due to the electromagnetic interaction of ultrarelativistic heavy ions which will be available at RHIC and LHC colliders. Thirdly, in order to evaluate the probability of formation of very hot nuclei, we calculate the distributions in excitation energies of the residual nuclei formed after completion of the cascade stage of the reaction. 


\section{Electromagnetic dissociation of nuclei}

\subsection{Preliminary remarks}

The process of electromagnetic dissociation is known for a long time. Since only relatively low beam energies were available, mainly the nuclear evaporation and fission processes were studied up to now. In refs. [8]-12] the interaction of low-Z ions $\left({ }^{16} \mathrm{O},{ }^{28} \mathrm{Si}^{32} \mathrm{~S}\right)$ with energies $0.7-200 \mathrm{~A} \mathrm{GeV}$ was investigated via the projectile fragmentation in nuclear emulsions or on different targets $(\mathrm{H}, \mathrm{C}, \mathrm{Al}, \mathrm{Cu}, \mathrm{Ag}, \mathrm{Sn}, \mathrm{Pb})$. The electromagnetic dissociation of target nuclei has been also studied experimentally [13]-[16], mainly via the measurement of $\gamma$-activity of target fragments.

The electromagnetic interaction of high-Z ultrarelativistic heavy ions (with Lorentz factors $\gamma \gg 1$ ) has attracted recently a special attention in connection with the storage of the relativistic heavy-ion beams. It turned out that the cross section of electromagnetic dissociation of nuclei, $\sigma_{E D}$ becomes comparable with or even larger than the total nuclear cross section. The first observation of $\sigma_{E D}$ values above 1 barn was reported for the interaction of ${ }^{139} \mathrm{La}$ projectiles with a ${ }^{197} \mathrm{Au}$ target [14].

There are some estimates for the cross sections expected for the planned relativistic heavy-ion colliders: RHIC at Brookhaven $(\sqrt{s}=200 \mathrm{~A} \mathrm{GeV})$ and LHC at CERN $(\sqrt{s}=6.5 A \mathrm{TeV})$. As shown by Baur and Bertulani 17 for ${ }^{238} \mathrm{U}$ on ${ }^{238} \mathrm{U}$ and Mercier et al. [13] for ${ }^{197} \mathrm{Au}$ on ${ }^{197} \mathrm{Au}$, at RHIC energy $\sigma_{E D}$ reach values of 40 and $23.8 \mathrm{~b}$, respectively, with the corresponding nuclear cross sections of 6.9 and $6.1 \mathrm{~b}$. This means that the lifetime of stored beams will be greatly affected by the ED process. Also the particle production in ED will represent a significant background in experiments on future heavy-ion colliders such as RHIC and LHC.

The nature of a photonuclear interaction depends on the de Broglie wavelength $\lambda=\hbar / p$ of a virtual photon. At $E_{\gamma} \leq 40 \mathrm{MeV}, \lambda$ is comparable with the nuclear size and an excitation of the whole nucleus in the form of a Giant Dipole Resonance (GDR) is most important in this region. In the energy range $40 \leq E_{\gamma} \leq 140 \mathrm{MeV}$ (below the pion production threshold), $\lambda$ is comparable to the internucleonic distance, and therefore the absorption of a photon by a neutron-proton pair should be the main reaction mechanism. At higher energies, when $\lambda$ becomes smaller than the nucleon radius, photons interact mainly with individual nucleons. This process is known as the photoproduction of hadrons. It is followed by the emission of fast nucleons and pions from the nucleus. In present work the photoabsorption process on a nucleus is considered in the framework of the IntraNuclear Cascade model (INC) for photonuclear reactions [18].

Although soft photons (i.e. with energy $E_{\gamma} \leq 100 \mathrm{MeV}$ ) dominate in the virtual photon spectrum, it extends up to several $\mathrm{GeV}$ for RHI with $\gamma \sim 10-$ 100. Most theoretical works on electromagnetic disintegration [17]-[21] study the 
excitation of GDR through the absorption of one or more virtual photons prior to the de-excitation stage. Less efforts have been made to understand the nature of ED induced by the virtual photons from the high-energy part of the spectrum. We know the only calculation of inclusive pion production cross section 19 and the only experimental observation of pions in ED [12], unfortunately with a very poor statistics. In the case of multiple pion photoproduction the nucleus can absorb a large amount of energy, that, in its turn, may result not only in the emission of one or two nucleons or fission, but also in a multifragment break-up of the nucleus.

\subsection{Description of the model}

Since a relativistic particle spends a short time $\Delta t$ near the collision partner, the virtual photon spectrum will contain all frequencies up to a maximum one of the order $\omega_{\max } \sim 1 / \Delta t$. The magnitude of the pulse is proportional to the square of the charge. Both maximum frequency and magnitude depend on the value of impact parameter $b$ [4].

According to the Weizsäcker-Williams method the spectrum of virtual photons from a stationary target of charge $Z_{t}$ as seen by a projectile moving with velocity $\beta=v / c$ is obtained by the integration over impact parameters $b>b_{\min }$ and is expressed as [4]:

$$
n\left(E_{\gamma}\right)=\frac{2 \alpha Z_{t}^{2}}{\pi \beta^{2} E_{\gamma}}\left(x K_{0}(x) K_{1}(x)-\frac{\beta^{2}}{2} x^{2}\left(K_{1}^{2}(x)-K_{0}^{2}(x)\right)\right) .
$$

Where $K_{0}$ and $K_{1}$ are the modified Bessel functions of zero and first order, $x=E_{\gamma} b_{\min } /(\gamma \beta \hbar c), b_{\min }$ is the minimal value of the impact parameter which corresponds to the onset of nuclear interaction. With the curvature correction this value can be estimated as

$$
b_{\text {min }}=r_{0}\left(A_{p}^{1 / 3}+A_{t}^{1 / 3}-X\left(A_{p}^{-1 / 3}+A_{t}^{-1 / 3}\right)\right),
$$

where $r_{0}=1.34 \mathrm{fm}, A_{p}$ and $A_{t}$ are the nuclear numbers in projectile and target nuclei, respectively, and the curvature parameter $X=0.75$ [13. The cross section of ED for the projectile of mass $A_{p}$ can be calculated as:

$$
\sigma_{E D}=\int_{0}^{\infty} n\left(E_{\gamma}\right) \sigma_{A_{p}}\left(E_{\gamma}\right) d E_{\gamma}
$$

where $\sigma_{A_{p}}\left(E_{\gamma}\right)$ is the appropriate photoabsorption cross section measured for the projectile nucleus with real photons [22] or calculated by a model.

A realistic estimation of $\sigma_{E D}$ can be done as follows. In the GDR and quasideuteron regions, $E_{\gamma} \leq 140 \mathrm{MeV}$, one can utilize the tabulated values of $\sigma_{A_{p}}\left(E_{\gamma}\right)$. Above the pion production threshold a universal behaviour of $\sigma_{A_{p}}\left(E_{\gamma}\right)$ 
can be used since the ratio $\sigma_{A_{p}}\left(E_{\gamma}\right) / A_{p}$ depends weakly on $A_{p}$ up to $E_{\gamma}=2-3$ $\mathrm{GeV}$. Having the data for one nucleus one can calculate the cross section for other nuclei [23]. In this region, the excitation of numerous baryonic resonances (mainly $\Delta(1232), N^{\star}(1520), N^{\star}(1680)$ ) provides a prominent variation of the photoabsorption cross section, even though most of the resonances suffer damping inside the nucleus [24]. Above $E_{\gamma} \sim 3 \mathrm{GeV}$ the ratio $\sigma_{A_{p}}\left(E_{\gamma}\right) / A_{p}$ becomes lower for heavy nuclei due to the well-known shadowing effect [22]. This effect, which might be explained by the hadronic component of the photon wave function, is not very strong, of the order of $20 \%$, even for heaviest nuclei.

Using the Monte Carlo technique, we have first simulated the energy $E_{\gamma}$ of a virtual photon distributed according to the product $n\left(E_{\gamma}\right) \sigma_{A_{p}}\left(E_{\gamma}\right)$, which is proportional to the probability of the electromagnetic interaction. This function is plotted in Fig. 1 for the cases of RHI collisions considered in the paper. As seen from Fig. 1, the probability to have a photonuclear reaction above the pion production threshold is essentially enhanced for RHI beams with $\gamma \gg 100$. The second step is the nuclear photoabsorption process which is treated on the basis of the IntraNuclear Cascade model (INC).

In ref. [5] we have already shown that the INC model can be used for the description of reactions leading to the nuclear multifragmentation. This approach can be easily generalized for the photonuclear processes. At high photon energies many hadrons are produced inside a nucleus in the course of a cascade process. Due to the knock-out of the intranuclear nucleons by fast nucleons and pions, the slower particles pass through a lower density region undergoing less rescatterings, the so-called trawling effect. In the present calculations in addition to the standard version of the INC model we use also the version with trawling, which takes into account the local depletion of nuclear density during the development of the intranuclear cascade. As was shown in [25, 26], the trawling effect is important for a realistic description of reactions at projectile energies above several $\mathrm{GeV}$. The annihilation of energetic antiprotons is an example of the processes where heating of nuclei by a multi-pion system takes place. Recently it was found [27] that the distributions of excitation energies of residual nuclei produced in this process are in a good agreement with predictions of the INC model with trawling. In the present paper the calculations with the two INC versions can be considered as a measure of uncertainty in describing the cascade stage.

The model of photonuclear reactions [18] used below takes into account quasideuteron absorption of virtual photons as well as the hadron production on intranuclear nucleons. We disregard the multiple excitation processes with the absorption of more than one photon prior to the de-excitation stage. As shown by Llope and Braun-Munzinger [21], the single photon absorption dominates at beam energies above $\sim 10 \mathrm{~A} \mathrm{GeV}$. Our estimates analogous to those made in ref. [2] show that the probabilities for absorbing simultaneously two or three photons with energies above $0.4 \mathrm{GeV}$ are less than $9 \%$ and $1.6 \%$, respectively, even for $\mathrm{Pb}+\mathrm{Pb}$ collisions at LHC energies. 
The channels of the hadron photoproduction on a nucleon which are taken into account in the model are listed in Table 1. The two-body channel $\gamma N \rightarrow \pi N$ dominates up to $E_{\gamma} \sim 0.5 \mathrm{GeV}$, while at energies $0.5 \leq E_{\gamma} \leq 2 \mathrm{GeV}$ the channels $\gamma N \rightarrow 2 \pi N$ and $\gamma N \rightarrow 3 \pi N$ play a major role. However in this energy region the contribution of two-body subchannels $\gamma N \rightarrow \pi B^{\star}$ and $\gamma N \rightarrow M^{\star} N$ ( $B^{\star}$ and $M^{\star}$ are baryon and meson resonances, respectively) is also prominent. Finally, when the photon energy reaches several $\mathrm{GeV}$, the multiple pion production becomes most important. A large number $(\sim 80)$ of many-body subchannels contribute to this process. As shown in ref. [18], the INC model describes well the available data on the meson and proton production on nuclei by quasi-monochromatic photons up to energies $E_{\gamma} \sim 10 \mathrm{GeV}$, that covers the main part of the total cross-section of ED reactions.

Excited residual nuclei are formed after the completion of the intranuclear cascades. The ensemble of residual nuclei is characterized by wide distributions in excitation energy, $E^{\star}$, nucleon, $A$, and proton, $Z$, numbers. The decay of the residual nuclei is described by the Statistical Multifragmentation Model (SMM) [5]. The SMM assumes that a hot nucleus expands to a "freeze-out" volume where it splits into primary hot fragments and nucleons in thermal equilibrium. The break-up channels are constrained by the total mass, charge and energy of the system. All fragments (and nucleons) are considered as Boltzmann particles while Fermi-gas approximation is used for their internal excitation. The probabilities of different break-up channels are calculated microcanonically according to their statistical weights. After primary break-up excited fragments propagate independently under mutual Coulomb field and undergo secondary decay described by evaporation, Fermi-break-up or fission depending on their mass and excitation energy. The simulation of the whole process is performed by the Monte Carlo method.

The main conclusions of the SMM are as follows. The decay of a residual nucleus is determined by its excitation energy per nucleon $E^{\star} / A$. For $E^{\star} / A<2$ $\mathrm{MeV}$, the de-excitation goes through the successive emission of particles from the compound nucleus (evaporation), or its fission. When the excitation energy of the nucleus exceeds about a half its total binding energy $\left(E^{\star} / A>4 \mathrm{MeV}\right)$, the explosive multifragment break-up dominates. In the transition region $(2 \leq$ $\left.E^{\star} / A \leq 4 \mathrm{MeV}\right)$ the both decay processes coexist. At $E^{\star} / A>10 \mathrm{MeV}$ the system breaks up into nucleons and lightest clusters (vaporization).

This hybrid approach combining the INC and SMM models is quite successful in explaining the fragmentation and multifragmentation data (see examples in refs. [5, 25]). However, as was pointed out in ref. [28], there are signs of early particle emission which accompany the after-cascade evolution of the residual nuclei towards the point of fragment separation at freeze-out. Therefore thermal excitation energies and masses of thermalized systems at multifragmentation could be smaller than calculated with the INC model. From our previous experience [5] we can approximately estimate an uncertainty introduced by this effect as no more 
than factor 2 in inclusive yields of fragments.

\subsection{Analysis of existing data}

With this combined approach we are able to calculate the yields of different isotopes in the ED process and compare them with existing experimental data. The isotope yields were measured in several experiments [9, 12]. As it is seen from Fig. 2, a wide range of isotopes is produced in the disintegration of light nuclei. Both versions of the INC model show a satisfactory agreement for 200A $\mathrm{GeV}$ ions, but for lower ion energy $(14.5 \mathrm{~A} \mathrm{GeV})$ the agreement is poor. Since our model is designed specially for RHI with $\gamma \gg 10$, we consider these results as acceptable.

In the emulsion experiments [9, 12 each decay mode into charged particles can be measured, and its fraction to the total number of observed projectile dissociation events can be determined. The calculated fraction of events in different groups of disintegration channels is given in Table 2. Taking into account that different experiments give different values, we conclude that the model describes these data fairly well.

As already mentioned, the decay mechanism of a residual nucleus depends on its excitation energy per nucleon. It is seen from Fig. 3 that the distribution of the excitation energies is very broad and tightly connected with the mass number of the initial nucleus and the projectile energy. The lighter nucleus $\left({ }^{16} \mathrm{O}\right.$ in comparison with ${ }^{32} \mathrm{~S}$ ) receives higher excitation energy per nucleon. For all reactions shown in Fig. 3 only a limited fraction of residual nuclei excited by virtual photons receives an excitation energy per nucleon sufficient for multifragmentation. However their absolute yields are large in comparison with those obtained in usual

nuclear reaction. It is the multiple pion production process that is responsible for production of heavy nuclei with $E^{\star} / A$ exceeding $2 \mathrm{MeV}$.

\section{Predictions for ultrarelativistic heavy-ion col- lisions}

As the above discussion shows, the most interesting ED processes should be expected in ultrarelativistic heavy-ions collisions at RHIC or LHC colliders [29]. The relative Lorentz factor $\gamma$ is about $2 \cdot 10^{4}$ for RHIC and $2 \cdot 10^{7}$ for LHC energies. The calculated cross sections $\sigma_{E D}$ for the ED process are given in Table 3 together with the nuclear fragmentation cross section $\sigma_{n u c}$ to be used for comparison. It is assumed that $\sigma_{n u c}$ does not depend on the projectile energy in the multi-GeV region and can be parametrized as [30]:

$$
\sigma_{n u c}=59\left(A_{p}^{1 / 3}+A_{t}^{1 / 3}-0.83\right)^{2}(m b)
$$


The $\sigma_{E D}$ is obtained by expression (3), where the integration covers all energies of virtual photons. One can estimate the contribution, $\sigma_{E D}^{\Delta}$, to the ED cross section coming from the photoabsorption in the $\Delta$-resonance region by limiting integration to photon energies between 0.14 and $0.4 \mathrm{GeV}$. The contribution from multiple pion production, $\sigma_{E D}^{M \pi}$, is obtained by integrating over photon energies above $0.4 \mathrm{GeV}$. The value $\sigma_{E D}^{I M F}$ is defined as a cross section to produce Intermediate Mass Fragments (IMF) with charges specified in Table 3: $Z=3-5$, $3-10$ or $3-30$, depending on the mass of the projectile which undergoes the multifragmentation.

As seen from Table of the model slightly overestimates the experimental $\sigma_{E D}$ values. One should bear in mind, however that $\sigma_{E D}$ is very sensitive to the details of the total photoabsorption cross section, $\sigma_{A_{p}}\left(E_{\gamma}\right)$, particularly in the GDR region. More accurate data for this cross section are certainly needed. The calculations show that the ED process becomes important already for heavy-ion collisions at CERN SpS $\left(160 \mathrm{~A} \mathrm{GeV}{ }^{208} \mathrm{~Pb}\right.$ on $\left.{ }^{208} \mathrm{~Pb}\right)$ which presently are under investigations.

Considering the values presented in the last two rows of Table 3, one can note that $\sigma_{E D}^{M \pi}$ exceeds $\sigma_{E D}^{\Delta}$ at RHIC and LHC energies and these two processes are responsible for $30-40 \%$ of the ED cross section. This proves that the pion photoproduction processes must be taken into consideration. For $\mathrm{Pb}+\mathrm{Pb}$ case $\sigma_{E D}$ is more than 10 times larger than $\sigma_{n u c}$. Even $\sigma_{E D}^{I M F}$ given by our model, turns out to be comparable or even greater than $\sigma_{n u c}$, that makes it possible to measure the electromagnetically induced multifragmentation of ultrarelativistic heavy ions.

In Fig. 1 we show predicted yields of fragments produced in the ED process. It is seen that the total cross-sections are by one order of magnitude higher than the ones observed in traditional nuclear processes. At LHC energies (bottom part) $\sigma(Z)$-distribution is enriched by intermediate mass fragments. The isotope yields are shown separately for singe and multiple pion production. Photons with energies in the $\Delta$-resonance region provide only a moderate nuclear excitation. In this case the de-excitation goes mainly through the emission of several nucleons (the left and right parts of Fig. (1) or fission (the central part). The absorption of a high-energy photon leads to a multiple pion production and, consequently, to a higher excitation of a nucleus. This results in the deep disintegration of the nucleus and multiple production of nuclear fragments with a wide mass distribution.

The average pion multiplicity, $n_{\pi}$, calculated over the whole region of virtual photon energies is not expected to be large, since the contribution from multiple pion photoproduction is not dominating in the ED process. Our calculations give $n_{\pi} \sim 1$ even for LHC energies, that is much lower than mean $n_{\pi}$ in nuclear collisions. But this is compensated by their large yields and broad multiplicity distributions. Inclusive cross sections of proton emission and pion production in the ED process are given in Table 6 . As one can see, these cross sections are 
predicted to be up to ten times larger than the nuclear cross sections.

\section{Conclusions}

It is demonstrated that the electromagnetic dissociation of nuclei gives a dominant contribution to the total cross section in ultrarelativistic heavy ion collisions. The fragmentation cross-section for RHIC and LHC beams exceeds considerably the standard nuclear cross sections. This means that the electromagnetic dissociation of ultrarelativistic beams on the residual gas should be considered seriously as a factor reducing the lifetime of the beams in RHIC or LHC colliders [29]. Its contribution to the spectra and multiplicities of particles must be considered in the planned experiments.

The electromagnetic fields generated by ultrarelativistic heavy ions are so strong that one can expect qualitatively new phenomena in these collisions. Nuclear multifragmentation is only one example of rich possibilities.

The long-range Coulomb forces could lead to a new type of collective motion. For example, instead of radial flow of nuclear matter caused by its compression in central nucleus-nucleus collisions [5] one may expect a perpendicular flow caused by tidal Coulomb forces. The Weizsäcker-Williams method of virtual photons might be not fully adequate to study these collective phenomena, especially at small impact parameters. We consider the present work only as a starting point for future studies.

We are grateful to J.J.Gaardhøje and L.M.Satarov for useful discussions. I.N.M is grateful to the Carlsberg Foundation for the financial support. A.S.B and I.A.P. thank the Niels Bohr institute for the warm hospitality and financial support. The work was supported partially by the Danish Natural Science Research Council and INTAS, grant 93-1560.

\section{References}

[1] A.Winther and K.Alder, Nucl. Phys. A319, 518 (1979).

[2] C.A.Bertulani and G.Baur, Phys. Reports 163, 299 (1988).

[3] A.C.Vasconcellos Gomes and C.A.Bertulani, Nucl. Phys. A517, 639 (1990).

[4] J.D.Jackson, Classical electrodynamics, 2nd ed., Wiley, New York, 1975.

[5] J.P.Bondorf, A.S.Botvina, A.S.Iljinov, I.N.Mishustin and K.Sneppen, Phys. Reports 257, 133 (1995).

[6] D.H.E.Gross, Rep. Progr. Phys. 53, 605 (1990).

[7] N.Baron and G.Baur, Phys. Rev. C 48, 1999 (1993). 
[8] C.Brechtmann and W.Heinrich, Z. Phys. A330, 407 (1988); A331, 463 (1988).

[9] G.Baroni, V.Bisi, A.C.Breslin, D.H.Davis, S.Dell'Uomo, S.Di Liberto, P.Giubellino, G.Grella, K.Hoshino, M.Kazuno, M.Kobayashi, K.Kodama, A.Marzari-Chiesa, M.A.Mazzoni, F.Meddi, M.T.Muciaccia, K.Niu, L.Ramello, G.Romano, G.Rosa, C.Sgarbi, H.Shibuya, S.Simone, D.N.Tovee, N.Ushida, C.Wilkin and S.K.C.Yuen, Nucl. Phys. A516, 673 (1990).

[10] G.Singh, K.Sengupta and P.L.Jain, Phys.Rev. C 41, 999 (1990).

[11] J.Barette et al. (E814 Collaboration), Phys. Rev. C 41, 1512 (1990).

[12] G.Singh, P.L.Jain, Z. Phys. A344, 73 (1992).

[13] M.T.Mercier, John C.Hill, F.K.Wohn, C.M.McCullough, M.E.Nieland, J.A.Winger, C.B.Howard, S.Renwick, and D.K.Matheis, Phys. Rev. C 33, 1655 (1986).

[14] John C.Hill, F.K.Wohn, J.A.Winger and A.R.Smith, Rhys. Rev. Lett. 60, 999 (1988).

[15] John C.Hill, F.K.Wohn, J.A.Winger, M.Khayat, K.Leininger and A.R.Smith, Phys. Rev. C 38, 1722 (1988).

[16] John C.Hill, F.K.Wohn, J.A.Winger, M.Khayat, M.T.Mercier and A.R.Smith, Phys. Rev. C 39, 524 (1989).

[17] G.Baur and C.A.Bertulani, Phys. Lett. B 174, 23 (1986).

[18] A.S.Iljinov, I.A.Pshenichnov, N.Bianchi, E.De Sanctis, V.Muccifora, M.Mirazita, P.Rossi, Nucl. Phys. A616, 575 (1997).

[19] C.A. Bertulani and G.Baur, Nucl. Phys. A458, 725 (1986).

[20] G.Baur and C.A.Bertulani, Phys. Rev. C 34, 1654 (1986).

[21] W.J.Llope and P.Braun-Munzinger, Phys. Rev. C 41, 2644 (1990).

[22] J.Ahrens, H.Borchert, K.H.Czock, H.B.Eppler, H.Gimm, H.Gundrim, M.Kröning, P.Rien, G.Sita Ram, A.Zieger and B.Ziegler, Nucl. Phys. A251, 479 (1975); J.Ahrens, Nucl. Phys. A446, 229c (1985).

[23] N.Bianchi, V.Muccifora, E.De Sanctis, A.Fantoni, P.Levi Sandri, E.Polli, A.R.Reolon, P.Rossi, M.Anghinolfi, P.Corvisiero, M. Ripani, M.Sanzone, M.Taiuti, and A.Zucchiatti, Phys. Rev. C 54, 1688 (1996). 
[24] L.A.Kondratyuk, M.I.Krivoruchenko, N.Bianchi, E.De Sanctis, V.Muccifora, Nucl. Phys. A579, 453 (1994).

[25] A.S.Botvina, A.S.Iljinov and I.N.Mishustin, Nucl. Phys. A507, 649 (1990).

[26] Ye.S.Golubeva, A.S.Iljinov and I.A.Pshenichnov, Phys. Atomic Nuclei 57, 2007 (1994).

[27] F.Goldenbaum, W.Bohne, J.Eades, T.v.Egidy, P.Figuera, H.Fuchs, J.Galin, Ye.S.Golubeva, K.Gulda, D.Hilscher, A.S.Iljinov, U.Jahnke, J.Jastrzebski, W.Kurcewicz, B.Lott, M.Morjean, G.Pausch, A.Peghaire, L.Pienkowski, D.Polster, S.Proschitzki, B.Quednau, H.Rossner, S.Schmid, W.Schmid, and P.Ziem, Phys. Rev. Lett. 77, 1230 (1996).

[28] A.S.Botvina and I.N.Mishustin, Phys. Lett. B294, 23 (1992).

[29] Conceptual design of the relativistic heavy ion collider RHIC, BNL 52195 UC-414, 1989, p.119; The Large Hadron Collider Accelerator Project, CERN/AC/93-03(LHC), 1993, p.79.

[30] Y.D.He, P.B.Price, Z. Phys. A348, 105 (1994). 
Table 1: Exclusive channels of the elementary $\gamma N$ interaction taken into account in the INC calculations.

\begin{tabular}{|c|c|}
\hline$\gamma p$-interaction & $\gamma n$-interaction \\
\hline $\begin{array}{l}\gamma p \rightarrow \pi^{+} n \\
\gamma p \rightarrow \pi^{0} p\end{array}$ & $\begin{array}{l}\gamma n \rightarrow \pi^{-} p \\
\gamma n \rightarrow \pi^{0} n\end{array}$ \\
\hline $\begin{array}{c}\gamma p \rightarrow \pi^{-} \Delta^{++} \\
\gamma p \rightarrow \pi^{0} \Delta^{+} \\
\gamma p \rightarrow \pi^{+} \Delta^{0}\end{array}$ & $\begin{array}{l}\gamma n \rightarrow \pi^{-} \Delta^{+} \\
\gamma n \rightarrow \pi^{0} \Delta^{0} \\
\gamma n \rightarrow \pi^{+} \Delta^{-}\end{array}$ \\
\hline $\begin{array}{c}\gamma p \rightarrow \eta p \\
\gamma p \rightarrow \omega p \\
\gamma p \rightarrow \rho^{0} p \\
\gamma p \rightarrow \rho^{+} n\end{array}$ & $\begin{array}{c}\gamma n \rightarrow \eta n \\
\gamma n \rightarrow \omega n \\
\gamma n \rightarrow \rho^{0} n \\
\gamma n \rightarrow \rho^{-} p\end{array}$ \\
\hline $\begin{array}{l}\gamma p \rightarrow \pi^{+} \pi^{-} p \\
\gamma p \rightarrow \pi^{0} \pi^{+} n\end{array}$ & $\begin{array}{l}\gamma n \rightarrow \pi^{+} \pi^{-} n \\
\gamma n \rightarrow \pi^{0} \pi^{-} p\end{array}$ \\
\hline $\begin{array}{c}\gamma p \rightarrow \pi^{0} \pi^{0} \pi^{0} p \\
\gamma p \rightarrow \pi^{+} \pi^{-} \pi^{0} p \\
\gamma p \rightarrow \pi^{+} \pi^{0} \pi^{0} n \\
\gamma p \rightarrow \pi^{+} \pi^{+} \pi^{-} n\end{array}$ & $\begin{array}{c}\gamma n \rightarrow \pi^{0} \pi^{0} \pi^{0} n \\
\gamma n \rightarrow \pi^{+} \pi^{-} \pi^{0} n \\
\gamma n \rightarrow \pi^{-} \pi^{0} \pi^{0} p \\
\gamma n \rightarrow \pi^{+} \pi^{-} \pi^{-} p\end{array}$ \\
\hline $\begin{array}{c}\gamma p \rightarrow i \pi N(4 \leq i \leq 8) \\
(35 \text { channels })\end{array}$ & $\begin{array}{c}\gamma n \rightarrow i \pi N(4 \leq i \leq 8) \\
(35 \text { channels })\end{array}$ \\
\hline
\end{tabular}


Table 2: Relative contribution of different decay modes for ED of the $200 \mathrm{~A} \mathrm{GeV}$ ${ }^{16} \mathrm{O}$ projectile in emulsion.

\begin{tabular}{|l|c|c|c|c|}
\hline \hline \multirow{2}{*}{ Decay mode } & \multicolumn{4}{|c|}{ Fraction (\% ) } \\
\cline { 2 - 5 } & Standard INC & INC with trawling & \multicolumn{2}{|c|}{ Experiments } \\
\cline { 4 - 5 } & and SMM & and SMM & {$[9]$} & {$[12]$} \\
\hline \hline${ }^{15} \mathrm{~N}+p$ & 50.4 & 46.7 & $56.08 \pm 3.93$ & $49.45 \pm 6.62$ \\
\hline${ }^{12} \mathrm{C}+\alpha$ & 36.0 & 43.2 & $25.58 \pm 2.61$ & $23.01 \pm 4.80$ \\
${ }^{12} \mathrm{C}+2 d$ & & & & \\
\hline $\begin{array}{l}{ }^{11} \mathrm{~B}+\alpha+p \\
{ }^{8} \mathrm{Be}+\alpha+2 d \\
{ }^{8} \mathrm{Be}+{ }^{7} \mathrm{Li}+p\end{array}$ & 8.6 & 6.26 & $4.42 \pm 1.10$ & $10.62 \pm 3.06$ \\
\hline $\begin{array}{l}{ }^{7} \mathrm{Li}+2 \alpha+p \\
{ }^{7} \mathrm{Li}+\alpha+2 d+p \\
{ }^{7} \mathrm{Li}+4 d+p\end{array}$ & 1.85 & 0.95 & $2.49 \pm 0.83$ & $4.42 \pm 1.98$ \\
\hline $\begin{array}{l}4 \alpha \\
3 \alpha+2 d\end{array}$ & 2.9 & 3.0 & $8.01 \pm 1.49$ & $12.39 \pm 3.31$ \\
$2 \alpha+4 d$ & & & & \\
$\alpha+6 d$ & & & & \\
\hline \hline
\end{tabular}


Table 3: Cross sections of the ED process (barn) calculated by the model for the present and future RHI beams. See the text for notations.

\begin{tabular}{|c|c|c|c|c|c|c|c|}
\hline \multirow[t]{2}{*}{ Reaction } & \multirow[t]{2}{*}{$\sigma_{n u c}$} & \multicolumn{2}{|r|}{$\sigma_{E D}$} & \multirow[t]{2}{*}{$\sigma_{E D}^{\Delta}$} & \multirow[t]{2}{*}{$\sigma_{E D}^{M \pi}$} & \multicolumn{2}{|c|}{$\sigma_{E D}^{I M F}$} \\
\hline & & theory & experiment & & & standard & trawling \\
\hline $\begin{array}{l}14.5 \mathrm{~A} \mathrm{GeV} \\
{ }^{28} \mathrm{Si} \text { on } \mathrm{Ag}\end{array}$ & 2.853 & "0.499 & $\begin{array}{c}0.383 \pm 0.037 \\
\text { |12 }\end{array}$ & 0.020 & 0.002 & $\begin{array}{c}0.039 \\
Z=3\end{array}$ & $\begin{array}{l}0.011 \\
-10\end{array}$ \\
\hline $\begin{array}{l}200 \mathrm{~A} \mathrm{GeV} \\
{ }^{16} \mathrm{O} \text { on } \mathrm{Ag}\end{array}$ & 2.445 & 0.844 & $\begin{array}{c}0.592 \pm 0.057 \\
\text { 12 }\end{array}$ & 0.110 & 0.080 & $\begin{array}{r}0.205 \\
Z=\end{array}$ & $\begin{array}{l}0.134 \\
-5 \\
\end{array}$ \\
\hline $\begin{array}{l}200 \mathrm{~A} \mathrm{GeV} \\
{ }^{32} \mathrm{~S} \text { on } \mathrm{Ag}\end{array}$ & 2.968 & 1.643 & $\begin{array}{c}1.680 \pm 0.080 \\
9]\end{array}$ & 0.213 & 0.145 & $\begin{array}{l}0.294 \\
\quad Z=3\end{array}$ & $\begin{aligned} & 0.086 \\
- & 10\end{aligned}$ \\
\hline $\begin{array}{c}160 \mathrm{~A} \mathrm{GeV} \\
{ }^{208} \mathrm{~Pb} \text { on }{ }^{208} \mathrm{~Pb}\end{array}$ & 7.164 & 40.00 & - & 3.070 & 1.662 & $\begin{array}{l}0.857 \\
\quad Z=3\end{array}$ & $\begin{aligned} & 0.091 \\
- & 30\end{aligned}$ \\
\hline $\begin{array}{c}100 \mathrm{~A}+100 \mathrm{~A} \mathrm{GeV} \\
{ }^{208} \mathrm{~Pb} \text { on }{ }^{208} \mathrm{~Pb}\end{array}$ & 7.164 & 101.15 & - & 11.50 & 16.74 & $\begin{array}{c}30.42 \\
Z=3\end{array}$ & $\begin{aligned} & 5.12 \\
&- 30 \\
&\end{aligned}$ \\
\hline $\begin{array}{l}3.2 \mathrm{~A}+3.2 \mathrm{~A} \mathrm{TeV} \\
{ }^{208} \mathrm{~Pb} \text { on }{ }^{208} \mathrm{~Pb}\end{array}$ & 7.164 & 202.7 & - & 23.92 & 42.46 & $\begin{array}{l}79.51 \\
Z=3\end{array}$ & $\begin{aligned} & 15.0 \\
&- 30 \\
&\end{aligned}$ \\
\hline
\end{tabular}

Table 4: Inclusive cross sections (barn) of proton emission and pion production in the electromagnetic dissociation of the projectile calculated by the INC model with trawling for the present and future RHI beams.

\begin{tabular}{|c|c|c|c|c|c|}
\hline \hline \multirow{2}{*}{ Reaction } & \multirow{2}{*}{$\sigma_{\text {nuc }}$} & \multicolumn{4}{|c|}{$\sigma_{E D}$} \\
\cline { 3 - 6 } & & $p$ & $\pi^{+}$ & $\pi^{-}$ & $\pi^{0}$ \\
\hline \hline $\begin{array}{c}14.5 \mathrm{~A} \mathrm{GeV} \\
28 \mathrm{Si} \text { on } \mathrm{Ag}\end{array}$ & 2.853 & 0.492 & 0.003 & 0.003 & 0.006 \\
\hline $\begin{array}{c}200 \mathrm{~A} \mathrm{GeV} \\
16\end{array}$ & 2.445 & 0.623 & 0.059 & 0.062 & 0.078 \\
\hline $\begin{array}{c}200 \mathrm{~A} \mathrm{GeV} \\
32\end{array}$ & 2.967 & 2.19 & 0.105 & 0.110 & 0.144 \\
\hline $\begin{array}{c}160 \mathrm{Ag} \mathrm{GeV} \\
208\end{array}$ & 7.164 & 9.72 & 1.04 & 1.68 & 2.00 \\
\hline $\begin{array}{c}100 \mathrm{~A}+100 \mathrm{~A} \mathrm{GeV} \\
208 \\
{ }^{208} \mathrm{~Pb} \text { on }{ }^{208} \mathrm{~Pb}\end{array}$ & 7.164 & 89.6 & 17.9 & 23.9 & 27.1 \\
\hline $\begin{array}{c}3.2 \mathrm{~A}+3.2 \mathrm{~A} \mathrm{TeV} \\
208\end{array}$ & 7.164 & 241.7 & 52.7 & 68.9 & 77.4 \\
\hline \hline
\end{tabular}




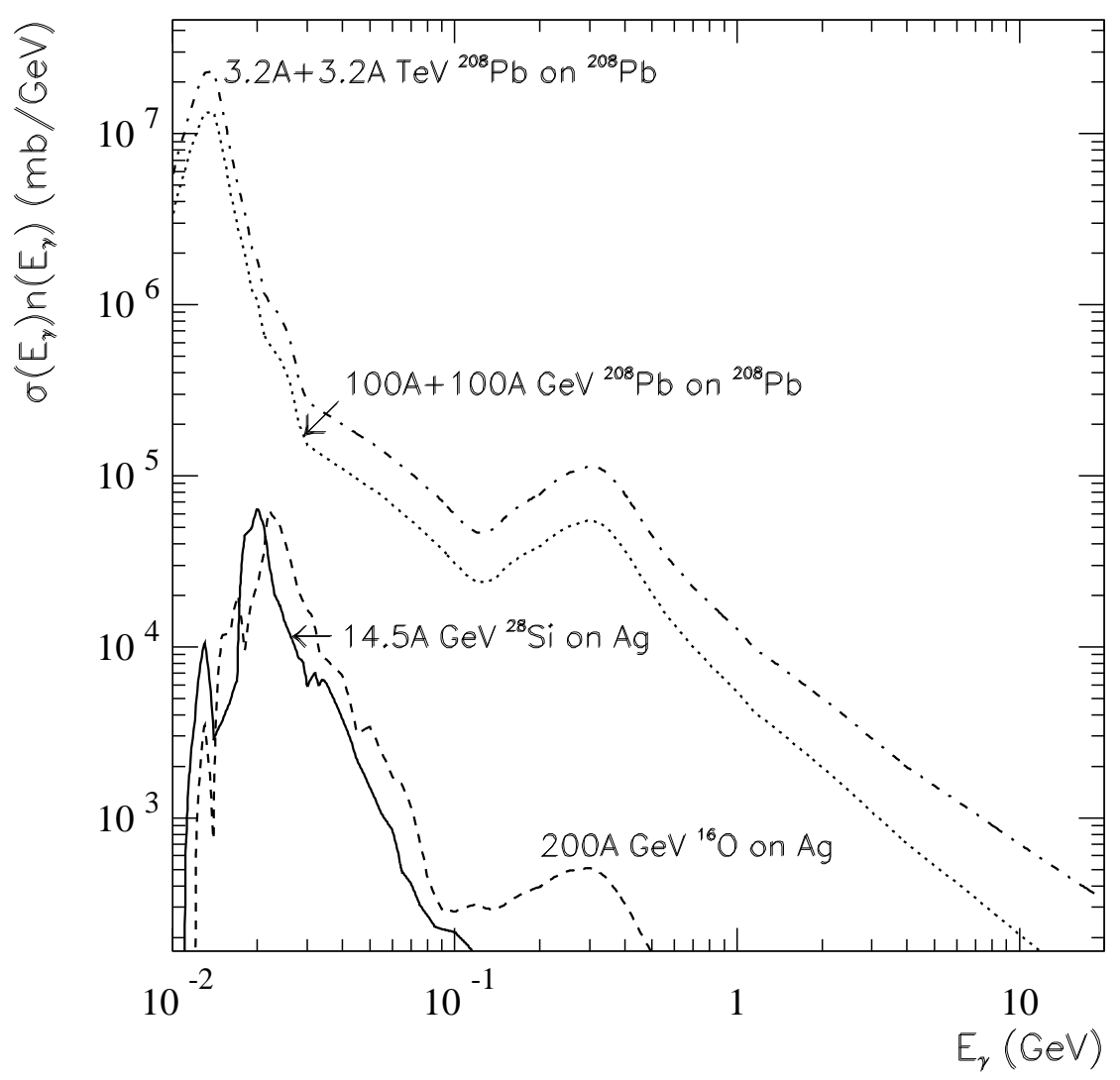

Figure 1: Product $n\left(E_{\gamma}\right) \sigma_{A_{p}}\left(E_{\gamma}\right)$ as a function of virtual photon energy $E_{\gamma}$. 

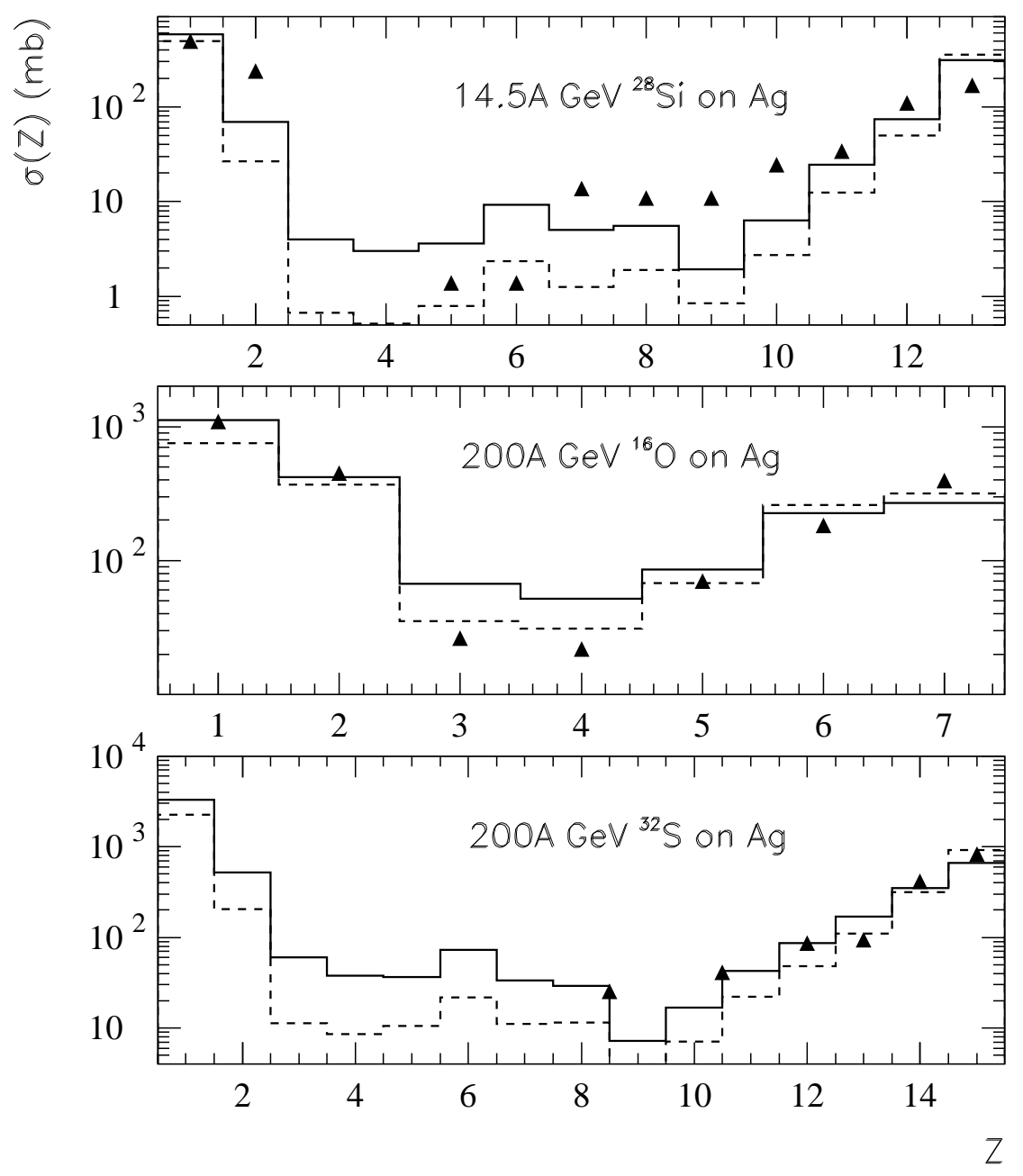

Figure 2: Inclusive cross sections of different isotope production in the electromagnetic dissociation of the projectile as a function of isotope charge $Z$. Triangles are the experimental data [9, 12]. Calculation results for two versions of the INC model, with and without trawling, are presented by dashed and solid histograms, respectively. 


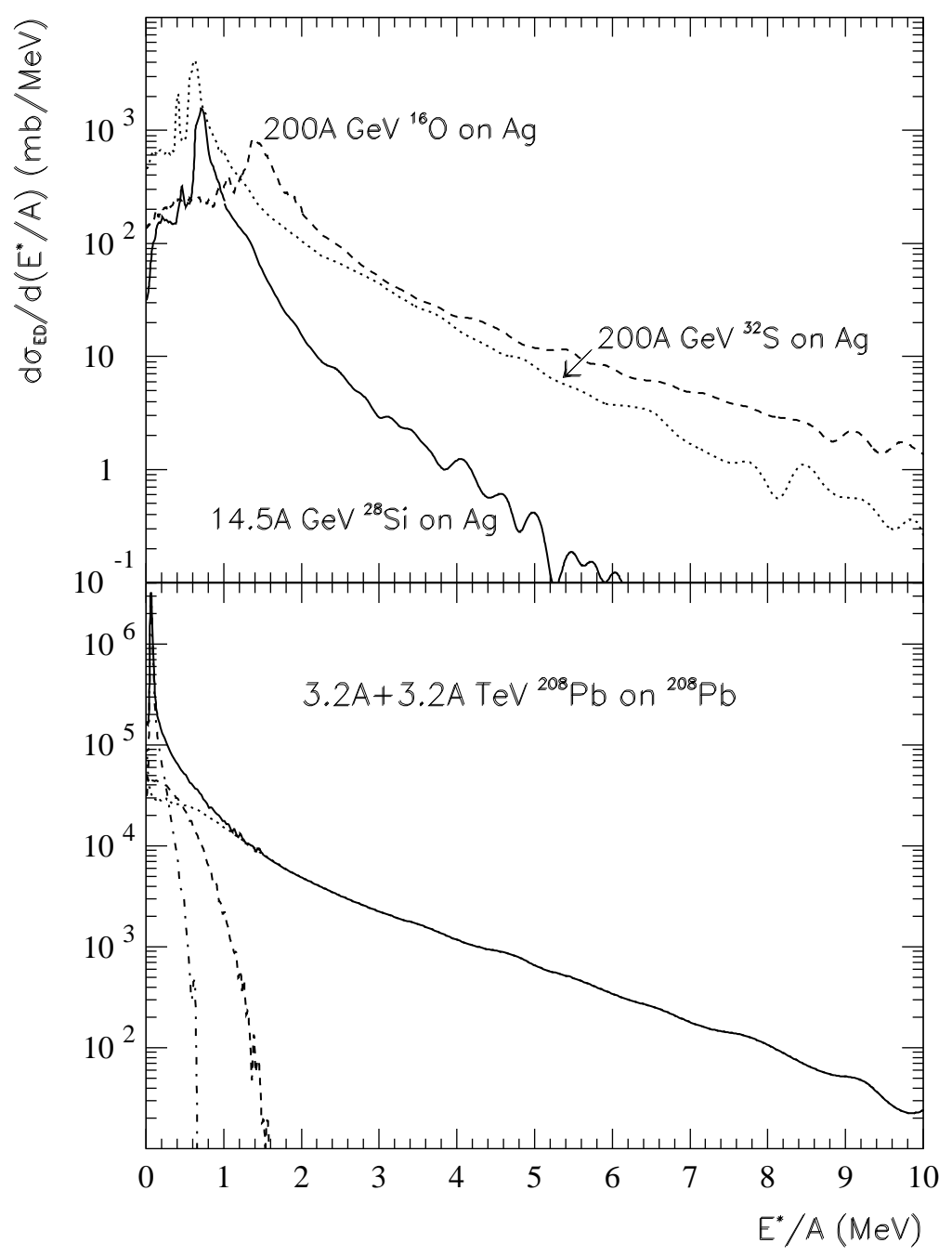

Figure 3: Distribution of excitation energies (per nucleon) of residual projectile nuclei produced in the electromagnetic interactions of the projectile and target nuclei. The calculations are presented for the INC model with trawling only. Results for $14.5 \mathrm{~A} \mathrm{GeV}{ }^{28} \mathrm{Si}$ on $\mathrm{Ag}, 200 \mathrm{~A} \mathrm{GeV}{ }^{16} \mathrm{O}$ and ${ }^{32} \mathrm{~S}$ on $\mathrm{Ag}$ are shown respectively by the solid, dashed and dotted lines in the top panel. Predictions for ${ }^{208} \mathrm{~Pb}+{ }^{208} \mathrm{~Pb}$ collisions at LHC energy are shown by the solid line in the bottom panel. Contributions from the processes of GDR, $\Delta$-isobar excitation and multiple pion production are shown by the dot-dashed, dashed and dotted lines, respectively. 


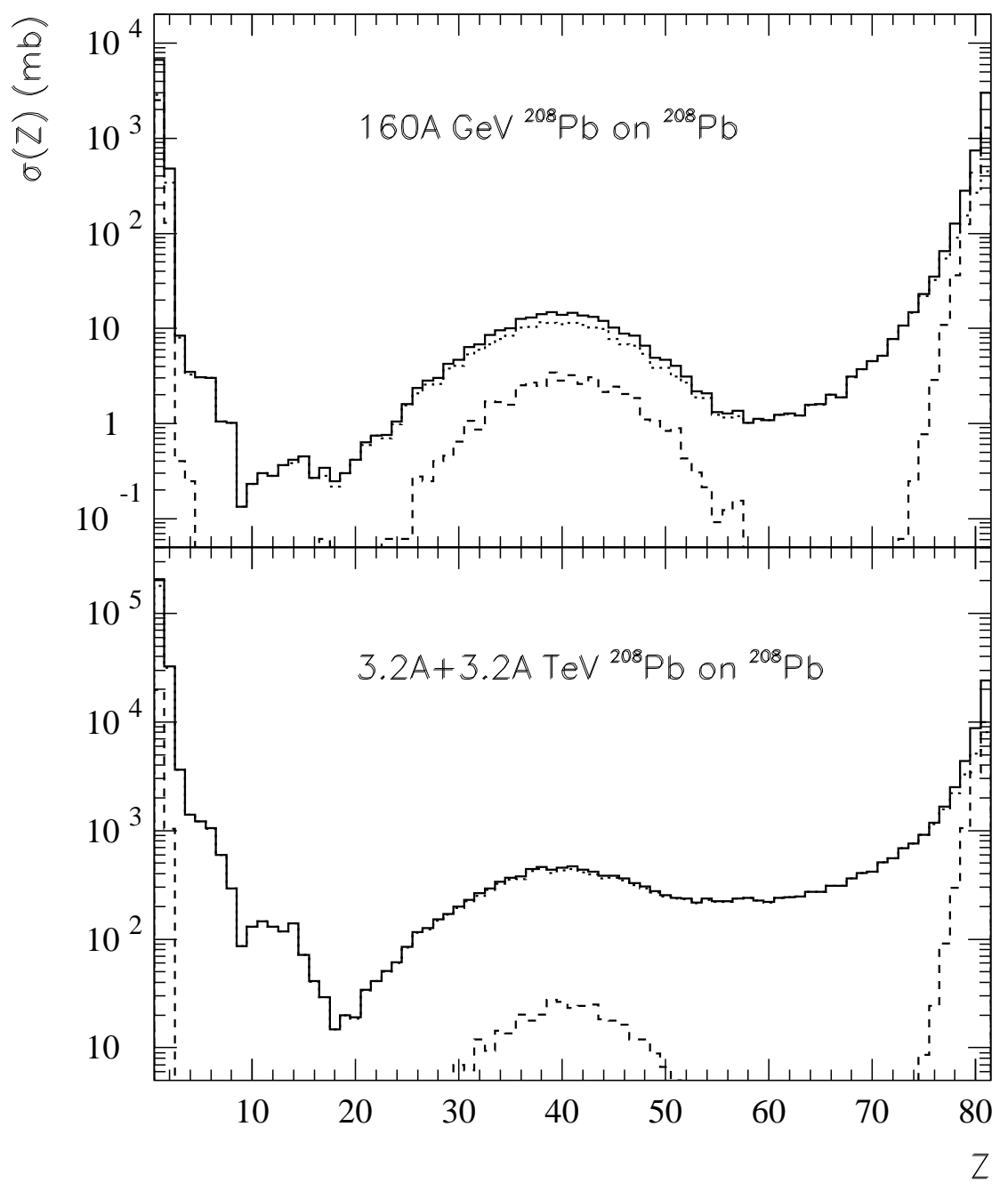

Figure 4: Inclusive cross sections of different isotope production in the electromagnetic dissociation of the projectile as a function of isotope charge $Z$. Predictions of the INC model with trawling are given. Contributions from the processes of $\Delta$-isobar excitation and multiple pion production are shown by the dashed and dotted histograms, respectively. 